\title{
THE LARGE DEVIATION PRINCIPLE FOR THE ON-OFF WEIBULL SOJOURN PROCESS
}

\author{
KEN R. DUFFY, ${ }^{*}$ National University of Ireland, Maynooth \\ ARTEM SAPOZHNIKOV, ${ }^{* *}$ Centrum voor Wiskunde en Informatica
}

\begin{abstract}
This article proves that the on-off renewal process with Weibull sojourn times satisfies the large deviation principle on a nonlinear scale. Unusually, its rate function is not convex. Apart from on a compact set, the rate function is infinite, which enables us to construct natural processes that satisfy the large deviation principle with nontrivial rate functions on more than one time scale.

Keywords: Weibull sojourn source; large deviation; nonlinear scale; nonconvex rate function

2000 Mathematics Subject Classification: Primary 60F10

Secondary 60G99
\end{abstract}

\section{Introduction}

Let $v(\cdot): \mathbb{R} \rightarrow \mathbb{R}$ be a nondecreasing function that diverges to $\infty$. A real-valued process $\left\{Z_{t}, t \in T\right\}$ (where $T$ is $\mathbb{N}$ or $\mathbb{R}$ ) satisfies the large deviation principle (LDP) on the scale $v(\cdot)$ with rate function $I: \mathbb{R} \rightarrow[0, \infty]$ if $I$ is lower semicontinuous and, for all Borel sets $B \subset \mathbb{R}$,

$$
-\inf _{x \in B^{\circ}} I(x) \leq \liminf _{t \rightarrow \infty} \frac{1}{v(t)} \log \mathrm{P}\left(Z_{t} \in B\right) \leq \limsup _{t \rightarrow \infty} \frac{1}{v(t)} \log \mathrm{P}\left(Z_{t} \in B\right) \leq-\inf _{x \in \bar{B}} I(x),
$$

where $B^{\circ}$ denotes the interior of $B$ and $\bar{B}$ denotes the closure of $B$. A rate function is good if its level sets $\{x: I(x) \leq \beta\}$ are compact for all $\beta<\infty$.

The averages $\left\{n^{-1} S_{n}=n^{-1} \sum_{i=1}^{n} Y_{i}\right\}$ of many real-valued processes are known to satisfy the LDP, including $\left\{Y_{i}\right\}$ being independent and identically distributed (i.i.d.) random variables or satisfying mixing conditions that are broad enough to encompass Doeblin recurrent Markov chains (see, e.g. [1]). If the tail of the distribution of $Y_{1}$ decays no slower than an exponential then the scale for the LDP is the number of summands, $n$, and, typically, the rate function is convex. If the summands have a semiexponential (Weibull) tail $\mathrm{P}\left(Y_{1}>y\right)=\exp \left(-y^{\alpha}\right)$, where $\alpha \in(0,1)$, then they satisfy the LDP on the scale $n^{\alpha}$ with the concave rate function given in Theorem 1 (see, e.g. [4]), which is finite for all arguments greater than or equal to the mean. (Fractional Brownian motion is an example of a process that satisfies the LDP on a nonlinear scale, but with a rate function that is convex and finite everywhere.) Rate functions that are not convex are interesting as one of the main tools in the theory of large deviations, the duality between the rate function and its Legendre-Fenchel transform (the scaled cumulant generating function) does not hold.

Here we prove the LDP for the on-off renewal process with Weibull sojourn times on the scale $t^{\alpha}$. Its rate function is not convex and, moreover, is only finite on a compact set. This

Received 27 March 2007; revision received 1 October 2007.

* Postal address: Hamilton Institute, National University of Ireland, Maynooth, Co. Kildare, Ireland.

** Postal address: Centrum voor Wiskunde en Informatica, Kruislaan 413, NL-1098SJ Amsterdam, Holland. 
provides a new example of a natural process whose properties cannot be deduced from GartnerEllis style theorems. Moreover, as its rate function is infinite off a compact set, we can readily construct simple processes that have nontrivial rate functions on more than one scale.

\section{Main result}

Let $\left\{\xi_{i}\right\}$ denote i.i.d. on-times and $\left\{\tau_{i}\right\}$ denote i.i.d. off-times, where an on-time follows an off-time which follows an on-time. Assume that, for $x>0$,

$$
\mathrm{P}\left(\xi_{1}>x\right)=\mathrm{P}\left(\tau_{1}>x\right)=\exp \left(-x^{\alpha}\right)
$$

where $\alpha \in(0,1)$, and denote

$$
\mu:=\mathrm{E}\left(\xi_{1}\right)=\alpha \int_{0}^{\infty} x^{\alpha-1} \exp \left(-x^{\alpha}\right) \mathrm{d} x=\Gamma\left(1+\alpha^{-1}\right)
$$

For each $n \in \mathbb{N}$ and $t \in \mathbb{R}_{+}$, define

$$
S_{n}^{\tau}:=\sum_{i=1}^{n} \tau_{i}, \quad S_{n}^{\xi}:=\sum_{i=1}^{n} \xi_{i}, \quad T_{n}:=S_{n}^{\tau}+S_{n}^{\xi}, \quad N_{t}:=\sup \left\{n: T_{n} \leq t\right\} .
$$

Theorem 1, below, is a well-known result for the partial sums of semiexponential distributed random variables (see, e.g. [3] or [4]).

Theorem 1. The process $\left\{S_{n}^{\xi} / n\right\}$ satisfies the LDP on the scale $n^{\alpha}$ with rate function

$$
I(x)= \begin{cases}(x-\mu)^{\alpha} & \text { if } x \geq \mu, \\ +\infty & \text { if } x<\mu .\end{cases}
$$

Here we are interested in an on-off process whose sojourn times are i.i.d. with semiexponential distribution. Define the on-time set $A:=\left\{s: s \in\left[T_{n}+\tau_{n+1}, T_{n+1}\right)\right.$ for some $\left.n\right\}$. The process of interest is the cumulative on-time prior to time $t$ :

$$
X_{t}:=\int_{0}^{t} \mathbf{1}_{A}(s) \mathrm{d} s, \quad \mathbf{1}_{A}(s)= \begin{cases}1 & \text { if } s \in A \\ 0 & \text { if } s \notin A\end{cases}
$$

The following theorem is the main result.

Theorem 2. (LDP for Weibull sojourn source.) The process $\left\{X_{t} / t\right\}$ satisfies the LDP in $\mathbb{R}$ on the scale $t^{\alpha}$ with good rate function

$$
J(x)= \begin{cases}(1-2 x)^{\alpha} & \text { if } x \in\left[0, \frac{1}{2}\right] \\ (2 x-1)^{\alpha} & \text { if } x \in\left[\frac{1}{2}, 1\right] \\ +\infty & \text { if } x \notin[0,1]\end{cases}
$$

The rate function defined in (2) is not convex; see, for example, Figure 1, in which we plot $J(x)$ versus $x$ for $\alpha=\frac{1}{2}$. As Gartner-Ellis theorems rely on convexity of the rate function, Theorem 2 cannot be deduced by that methodology. 


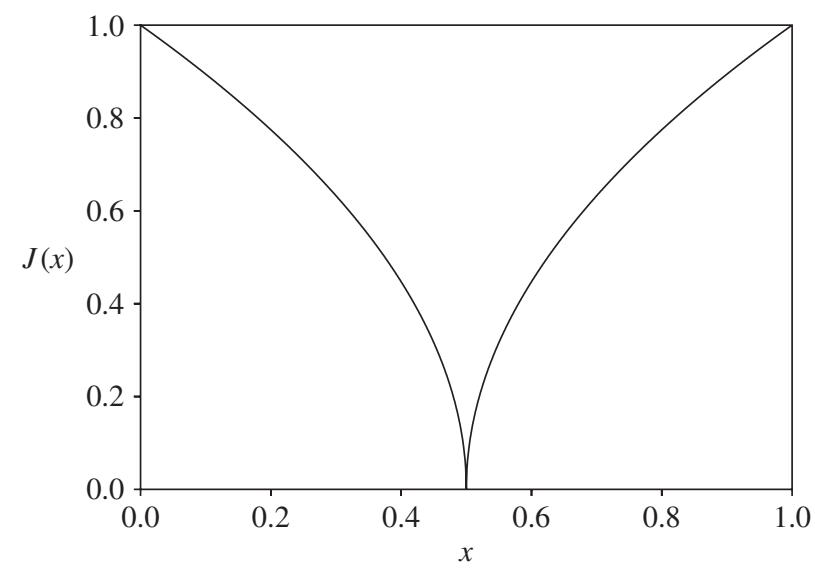

FIGURE 1: Rate function for $\alpha=\frac{1}{2}$.

Proof of Theorem 2. Let $B_{\varepsilon}(x)$ denote the open ball of radius $\varepsilon$ around $x$. Our approach to proving Theorem 2 is to show that the lower deviation function

$$
\lim _{\varepsilon \rightarrow 0} \liminf _{t \rightarrow \infty} \frac{1}{t^{\alpha}} \log \mathrm{P}\left(\frac{X_{t}}{t} \in B_{\varepsilon}(x)\right)
$$

and the upper deviation function

$$
\lim _{\varepsilon \rightarrow 0} \limsup _{t \rightarrow \infty} \frac{1}{t^{\alpha}} \log \mathrm{P}\left(\frac{X_{t}}{t} \in B_{\varepsilon}(x)\right)
$$

coincide for all $x$. Once the lower and upper deviation functions are shown to be equal, as $X_{t} / t$ takes values in the compact set $[0,1]$, the LDP follows from, for example, Theorem 4.1.11 of [2]. That the upper and lower deviation functions coincide follows from Theorems 3 and 4 , below, whose proofs can be found in Sections 3 and 4, respectively.

Theorem 3. For all $x \in \mathbb{R}$,

$$
\lim _{\varepsilon \rightarrow 0} \liminf _{t \rightarrow \infty} \frac{1}{t^{\alpha}} \log \mathrm{P}\left(\frac{X_{t}}{t} \in B_{\varepsilon}(x)\right) \geq-J(x) .
$$

Theorem 4. For all $x \in \mathbb{R}$,

$$
\limsup _{\varepsilon \rightarrow 0} \limsup _{t \rightarrow \infty} \frac{1}{t^{\alpha}} \log \mathrm{P}\left(\frac{X_{t}}{t} \in B_{\varepsilon}(x)\right) \leq-J(x) .
$$

Remark 1. (Concave rate functions.) Although $J$ is concave (where finite) on either side of its mean, the sum of two independent copies of $\left\{X_{t}\right\}$ satisfies the LDP with a rate function that is not concave on either side of its mean.

Remark 2. (Nontrivial large deviations on more than one scale.) We say that a rate function $I$ is nontrivial if (i) it is finite at more than a single point, and (ii) it is not 0 everywhere where it is finite. A rate function that is not nontrivial is called trivial. 
As we have constructed a process that satisfies the LDP on a sublinear scale, $t^{\alpha}$, with a rate function that is finite only on a compact interval, we can now construct natural processes that satisfy the LDP with nontrivial rate functions on more than one scale. This leads to the emergence of multiple fundamental time scales for the exponential decay of probability for this process.

We demonstrate this by considering an example constructed by the sum of the Weibull sojourn process with an independent Bernoulli process. First note that from Theorem 2 it is easy to show that $\left\{X_{t} / t\right\}$ also satisfies the LDP on the scale $t$, but with the trivial good rate function $J_{1}(x)=0$ if $x \in[0,1]$ :

$$
J_{1}(x)= \begin{cases}0 & \text { if } x \in[0,1] \\ +\infty & \text { if } x \notin[0,1]\end{cases}
$$

Next consider a Bernoulli process: let $\left\{Z_{n}\right\}$ be an i.i.d. sequence of random variables taking the values 0 and 1 with $\mathrm{P}\left(Z_{n}=0\right)=1-p$ and $\mathrm{P}\left(Z_{n}=1\right)=p$ for some $p \in(0,1)$. With $Y_{t}=\sum_{i=1}^{[t]} Z_{i}$, it is well known that $\left\{Y_{t} / t\right\}$ satisfies the LDP on the scale $t$ with the nontrivial good rate function

$$
H_{1}(x)= \begin{cases}x \log \left(\frac{x}{p}\right)+(1-x) \log \left(\frac{1-x}{1-p}\right) & \text { if } x \in[0,1] \\ +\infty & \text { if } x \notin[0,1]\end{cases}
$$

On the scale $t^{\alpha},\left\{Y_{t} / t\right\}$ satisfies the LDP with the trivial good rate function

$$
H(x)= \begin{cases}0 & \text { if } x=p \\ +\infty & \text { if } x \neq p\end{cases}
$$

As the rate functions $J$ and $H$ are both good and addition is continuous, by the contraction principle (see, e.g. [2, Theorem 4.2.1]), $\left\{\left(X_{t}+Y_{t}\right) / t\right\}$ satisfies the LDP on the scale $t^{\alpha}$ with the nontrivial good rate function

$$
K(x)=\inf _{y}\{J(y)+H(x-y)\}=J(x-p) .
$$

However, both $J_{1}$ and $H_{1}$ are also good rate functions, so that, by the contraction principle, $\left\{\left(X_{t}+Y_{t}\right) / t\right\}$ also satisfies the LDP on the scale $t$ with the nontrivial good rate function

$$
K_{1}(x)=\inf _{y}\left\{J_{1}(y)+H_{1}(x-y)\right\}= \begin{cases}H_{1}(x) & \text { if } x \in[0, p], \\ 0 & \text { if } x \in[p, 1+p], \\ H_{1}(x-1) & \text { if } x \in[1+p, 2] \\ +\infty & \text { otherwise }\end{cases}
$$

In Figure 2 we plot $K(x)$ and $K_{1}(x)$ for $p=\alpha=\frac{1}{2}$. Outside $[p, 1+p]$, large deviations occur on the scale $t$, but inside $[p, 1+p]$, large deviations occur nontrivially on the scale $t^{\alpha}$. Thus, for the sum of the Weibull sojourn process and an independent Bernoulli process, we have the following large deviation approximations for large $t$ : if $x \in[p, 1+p]$,

$$
\mathrm{P}\left(X_{t}+Y_{t} \approx x t\right) \approx \exp \left(-t^{\alpha} K(x)\right)
$$




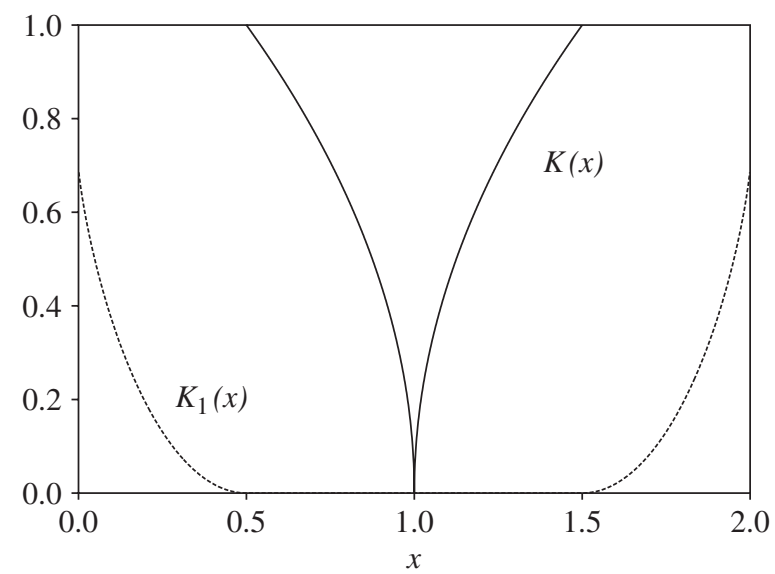

FIGURE 2: Rate functions for $\left\{\left(X_{t}+Y_{t}\right) / t\right\}$ with $\alpha=p=\frac{1}{2}$. The rate function $K(x)$ for the scale $t^{\alpha}$ is infinite outside $[0.5,1.5]$. The rate function $K_{1}(x)$ for the scale $t$ is infinite outside $[0,2]$ and is 0 inside $[0.5,1.5]$.

and if $x \in[0, p) \cup[1+p, 2]$,

$$
\mathrm{P}\left(X_{t}+Y_{t} \approx x t\right) \approx \exp \left(-t K_{1}(x)\right) .
$$

That is, $\left\{\left(X_{t}+Y_{t}\right) / t\right\}$ satisfies two nontrivial LDPs, with the probability decaying on a faster time scale outside $[p, 1+p]$.

\section{Proof of Theorem 3}

Recall the statement of Theorem 3: for all $x \in \mathbb{R}$,

$$
\lim _{\varepsilon \rightarrow 0} \liminf _{t \rightarrow \infty} \frac{1}{t^{\alpha}} \log \mathrm{P}\left(\frac{X_{t}}{t} \in B_{\varepsilon}(x)\right) \geq-J(x) .
$$

There are three cases to consider: $x=\frac{1}{2}, x \in\left[0, \frac{1}{2}\right)$, and $x \in\left(\frac{1}{2}, 1\right]$. If $x=\frac{1}{2}$, let $\varepsilon^{\prime}=\varepsilon$ and if $x \neq \frac{1}{2}$, let $\varepsilon^{\prime}=\min \left(\varepsilon,\left|x-\frac{1}{2}\right|\right)$. For any $n$, we have

$$
\begin{aligned}
\left\{\frac{X_{t}}{t}\right. & \left.\in B_{\varepsilon}(x)\right\} \\
& \supset\left\{\frac{X_{t}}{t} \in B_{\varepsilon^{\prime}}(x)\right\} \\
& \supset\left\{N_{t}=n, \frac{X_{t}}{t} \in B_{\varepsilon^{\prime}}(x)\right\} \\
& \supset\left\{T_{n}<t, T_{n}+\tau_{n+1}>t, \frac{X_{t}}{t} \in B_{\varepsilon^{\prime}}(x)\right\} \\
& \supset\left\{x-\frac{\varepsilon^{\prime}}{2}<\frac{S_{n}^{\xi}}{t}<x+\frac{\varepsilon^{\prime}}{2}, 1-x-\varepsilon^{\prime}<\frac{S_{n}^{\tau}}{t} \leq 1-x-\frac{\varepsilon^{\prime}}{2}, \tau_{n+1}>2 \varepsilon^{\prime} t+\mu\right\} .
\end{aligned}
$$

The final line is an inclusion as members of the set imply that $T_{n}=S_{n}^{\xi}+S_{n}^{\tau}<t, T_{n}+\tau_{n+1}>t$, and $X_{t} / t=S_{n}^{\xi} / t \in B_{\mathcal{E}^{\prime}}(x)$. As the three conditions in (3) correspond to independent events, 
we have, for any nondecreasing sequence $\left\{n_{t}\right\}$,

$$
\begin{aligned}
\liminf _{t \rightarrow \infty} \frac{1}{t^{\alpha}} \log \mathrm{P}\left(\frac{X_{t}}{t} \in B_{\varepsilon}(x)\right) \geq & \liminf _{t \rightarrow \infty} \frac{1}{t^{\alpha}} \log \mathrm{P}\left(\frac{S_{n_{t}}^{\xi}}{t} \in B_{\varepsilon^{\prime} / 2}(x)\right) \\
& +\liminf _{t \rightarrow \infty} \frac{1}{t^{\alpha}} \log \mathrm{P}\left(\frac{S_{n_{t}}^{\tau}}{t} \in B_{\varepsilon^{\prime} / 4}\left(1-x-\frac{3 \varepsilon^{\prime}}{4}\right)\right) \\
& +\liminf _{t \rightarrow \infty} \frac{1}{t^{\alpha}} \log \mathrm{P}\left(\tau_{n_{t}}>2 \varepsilon^{\prime} t+\mu\right) .
\end{aligned}
$$

For each $x \in \mathbb{R}$, define $\lfloor x\rfloor$ to be the greatest integer that is less than $x$. For $x=\frac{1}{2}$, we choose $n=n_{t}=\lfloor t / \mu\rfloor$; for $x \in\left[0, \frac{1}{2}\right)$, we choose $n=n_{t}=\left\lfloor t\left(x-\varepsilon^{\prime}\right) / \mu\right\rfloor$; and, for $x \in\left(\frac{1}{2}, 1\right]$, we choose $n=n_{t}=\left\lfloor t\left(1-x-\varepsilon^{\prime}\right) / \mu\right\rfloor$. As near identical arguments apply for all three cases, we shall only write out the proof for $x \in\left(\frac{1}{2}, 1\right]$. We apply the result of Theorem 1 to lower bound the first term on the right-hand side of (4), which gives

$$
\begin{aligned}
& \liminf _{t \rightarrow \infty} \frac{1}{t^{\alpha}} \log \mathrm{P}\left(\frac{S_{n_{t}}^{\xi}}{t} \in B_{\varepsilon^{\prime} / 2}(x)\right) \\
& \quad=\frac{\left(1-x-\varepsilon^{\prime}\right)^{\alpha}}{\mu^{\alpha}} \liminf _{n \rightarrow \infty} \frac{1}{n^{\alpha}} \log \mathrm{P}\left(\frac{S_{n}^{\xi}}{n} \in B_{\varepsilon^{\prime} \mu / 2\left(1-x-\varepsilon^{\prime}\right)}\left(\frac{\mu x}{1-x-\varepsilon^{\prime}}\right)\right) \\
& \quad \geq-\left(2 x-1+\frac{1}{2} \varepsilon^{\prime}\right)^{\alpha} .
\end{aligned}
$$

For the second term in (3), we again apply Theorem 1, which gives

$$
\begin{aligned}
\liminf _{t \rightarrow \infty} & \frac{1}{t^{\alpha}} \log \mathrm{P}\left(\frac{S_{n_{t}}^{\tau}}{t} \in B_{\varepsilon^{\prime} / 4}\left(1-x-\frac{3 \varepsilon^{\prime}}{4}\right)\right) \\
\quad= & \frac{\left(1-x-\varepsilon^{\prime}\right)^{\alpha}}{\mu^{\alpha}} \liminf _{n \rightarrow \infty} \frac{1}{n^{\alpha}} \log \mathrm{P}\left(\frac{S_{n}^{\tau}}{n} \in B_{\mu \varepsilon / 4\left(1-x-\varepsilon^{\prime}\right)}\left(\frac{\mu(1-x-3 \varepsilon / 4)}{1-x-\varepsilon^{\prime}}\right)\right) \\
& \geq-\frac{(1-x-\varepsilon)^{\alpha}}{\mu^{\alpha}} \inf \left\{I(a): a \in\left(\mu, \frac{\mu\left(1-x-\varepsilon^{\prime} / 2\right)}{1-x-\varepsilon^{\prime}}\right)\right\} \\
& =0,
\end{aligned}
$$

as $I(\mu)=0$. Finally, for the third term, from the Weibull distribution of $\tau$,

$$
\liminf _{t \rightarrow \infty} t^{-\alpha} \log \mathrm{P}\left(\tau>2 \varepsilon^{\prime} t+\mu\right)=-\left(2 \varepsilon^{\prime}\right)^{\alpha} .
$$

Hence, from the bound in (4) we have

$$
\liminf _{t \rightarrow \infty} \frac{1}{t^{\alpha}} \log \mathrm{P}\left(\frac{X_{t}}{t} \in B_{\varepsilon}(x)\right) \geq-\left(2 x-1+\frac{1}{2} \varepsilon^{\prime}\right)^{\alpha}-\left(2 \varepsilon^{\prime}\right)^{\alpha} .
$$

The result follows by taking $\varepsilon$ (and thus $\varepsilon^{\prime}$ ) to 0 .

\section{Proof of Theorem 4}

Recall the statement of Theorem 4: For all $x \in \mathbb{R}$,

$$
\lim _{\varepsilon \rightarrow 0} \limsup _{t \rightarrow \infty} \frac{1}{t^{\alpha}} \log \mathrm{P}\left(\frac{X_{t}}{t} \in B_{\varepsilon}(x)\right) \leq-J(x) .
$$


In order to prove this theorem we need Proposition 1, below, which will be proved using Lemmas 1 and 2 that appear later in this section.

Proposition 1. With $J$ defined as in (2), both $\left\{S_{N_{t}}^{\xi} / t\right\}$ and $\left\{S_{N_{t}}^{\tau} / t\right\}$ satisfy the LDP with good rate function $J(\cdot)$.

Once Proposition 1 is established, the upper bound on the upper deviation function for $\left\{X_{t} / t\right\}$ can be deduced from the following argument. First note that as $X_{t}$ is nondecreasing,

$$
S_{N_{t}}^{\xi} \leq X_{t} \leq t-S_{N_{t}}^{\tau}
$$

so that we have

$$
\begin{aligned}
& \quad \mathrm{P}\left(\frac{X_{t}}{t} \in B_{\varepsilon}(x)\right) \leq \mathrm{P}\left(\frac{X_{t}}{t}>x-\varepsilon\right) \leq \mathrm{P}\left(\frac{S_{N_{t}}^{\tau}}{t}<1-x+\varepsilon\right) \\
& \text { and } \mathrm{P}\left(\frac{X_{t}}{t} \in B_{\varepsilon}(x)\right) \leq \mathrm{P}\left(\frac{X_{t}}{t}<x+\varepsilon\right) \leq \mathrm{P}\left(\frac{S_{N_{t}}^{\xi}}{t}<x+\varepsilon\right) .
\end{aligned}
$$

Using these inequalities, we obtain

$$
\begin{aligned}
\lim _{\varepsilon \rightarrow 0} \limsup _{t \rightarrow \infty} \frac{1}{t^{\alpha}} \log \mathrm{P}\left(\frac{X_{t}}{t} \in B_{\varepsilon}(x)\right) \leq \min ( & \limsup _{t \rightarrow \infty} \frac{1}{t^{\alpha}} \log \mathrm{P}\left(\frac{S_{N_{t}}^{\xi}}{t}<x+\varepsilon\right), \\
& \left.\limsup _{t \rightarrow \infty} \frac{1}{t^{\alpha}} \log \mathrm{P}\left(\frac{S_{N_{t}}^{\tau}}{t}<1-x+\varepsilon\right)\right) .
\end{aligned}
$$

Employing the LDP upper bounds for $\left\{S_{N_{t}}^{\xi} / t\right\}$ and $\left\{S_{N_{t}}^{\tau} / t\right\}$ from Proposition 1, we see in the limit $\varepsilon$ tending to 0 that if $x<\frac{1}{2}$, the first term dominates and we obtain an upper bound of $-J(x)$. If $x>\frac{1}{2}$, the second term dominates and we obtain an upper bound of $-J(1-x)=-J(x)$. This completes the proof of Theorem 4 .

All that remains to do is to prove Proposition 1. As $\left\{S_{N_{t}}^{\xi}\right\}$ and $\left\{S_{N_{t}}^{\tau}\right\}$ are equal in distribution, we shall prove the result only for the former. To do this, we employ the same approach as described for Theorem 2. We will show that the lower and upper deviation functions coincide, i.e.

$$
\lim _{\varepsilon \rightarrow 0} \liminf _{t \rightarrow \infty} \frac{1}{t^{\alpha}} \log \mathrm{P}\left(\frac{S_{N_{t}}^{\xi}}{t} \in B_{\varepsilon}(x)\right)=\lim _{\varepsilon \rightarrow 0} \limsup _{t \rightarrow \infty} \frac{1}{t^{\alpha}} \log \mathrm{P}\left(\frac{S_{N_{t}}^{\xi}}{t} \in B_{\varepsilon}(x)\right) .
$$

By replacing $X_{t}$ with $S_{N_{t}}^{\xi}$ in the set inclusion (3), it can be seen that the arguments in the proof of Theorem 3 also show that

$$
-J(x) \leq \lim _{\varepsilon \rightarrow 0} \liminf _{t \rightarrow \infty} \frac{1}{t^{\alpha}} \log \mathrm{P}\left(\frac{S_{N_{t}}^{\xi}}{t} \in B_{\varepsilon}(x)\right) .
$$

Thus, it suffices to prove that

$$
\lim _{\varepsilon \rightarrow 0} \limsup _{t \rightarrow \infty} \frac{1}{t^{\alpha}} \log \mathrm{P}\left(\frac{S_{N_{t}}^{\xi}}{t} \in B_{\varepsilon}(x)\right) \leq-J(x) .
$$

Note that when $x=\frac{1}{2}$, the upper bound is obtained trivially by using 1 in place of the probability. We deduce the upper bound for $x \neq \frac{1}{2}$ from Lemmas 1 and 2, below, and by appealing to the principle of the largest term (see, e.g. [2, Lemma 1.2.15]). 
Lemma 1. (Large n.) For $x \in(0,1)$, define $\bar{x}:=\max (x, 1-x)$. If $x \in(0,1)$, for any $0<\varepsilon^{\prime}<\mu=\mathrm{E}\left(\tau_{1}\right)$, we have

$$
\lim _{\varepsilon \rightarrow 0} \limsup _{t \rightarrow \infty} \frac{1}{t^{\alpha}} \log \mathrm{P}\left(\frac{S_{N_{t}}^{\xi}}{t} \in B_{\varepsilon}(x), \frac{N_{t}}{t}>\frac{1-\bar{x}}{\mu-\varepsilon^{\prime}}\right)=-\infty .
$$

If $x=0$ or $x=1$ then, for any $0<\varepsilon^{\prime}<\mu$, we have

$$
\lim _{\varepsilon \rightarrow 0} \limsup _{t \rightarrow \infty} \frac{1}{t^{\alpha}} \log \mathrm{P}\left(\frac{S_{N_{t}}^{\xi}}{t} \in B_{\varepsilon}(x), \frac{N_{t}}{t}>\frac{\varepsilon}{\mu-\varepsilon^{\prime}}\right)=-\infty .
$$

Proof. For $x \in \mathbb{R}$, define $\lceil x\rceil$ to be the least integer that is greater than $x$. There are four cases to consider: $x=0, x=1, x \in\left(0, \frac{1}{2}\right)$, and $x \in\left(\frac{1}{2}, 1\right)$. We start with $x=0$. As $S_{n}^{\xi}$ is increasing in $n$, we have

$$
\left\{\frac{S_{N_{t}}^{\xi}}{t} \in B_{\varepsilon}(0), \frac{N_{t}}{t}>\frac{\varepsilon}{\mu-\varepsilon^{\prime}}\right\}=\left\{\frac{S_{N_{t}}^{\xi}}{t}<\varepsilon, \frac{N_{t}}{t}>\frac{\varepsilon}{\mu-\varepsilon^{\prime}}\right\} \subset\left\{S_{\left\lceil t \varepsilon /\left(\mu-\varepsilon^{\prime}\right)\right\rceil}^{\xi}<\varepsilon t\right\} .
$$

Applying the large deviations upper bound from Theorem 1 for this final sequence of sets, with $I$ being defined in (1), we obtain

$$
\begin{aligned}
\limsup _{t \rightarrow \infty} \frac{1}{t^{\alpha}} \log \mathrm{P}\left(S_{\left\lceil t \varepsilon /\left(\mu-\varepsilon^{\prime}\right)\right\rceil}^{\xi}<\varepsilon t\right) & =\frac{\varepsilon^{\alpha}}{\left(\mu-\varepsilon^{\prime}\right)^{\alpha}} \limsup _{n \rightarrow \infty} \frac{1}{n^{\alpha}} \log \mathrm{P}\left(\frac{S_{n}^{\xi}}{n}<\mu-\varepsilon^{\prime}\right) \\
& \leq-\frac{\varepsilon^{\alpha}}{\left(\mu-\varepsilon^{\prime}\right)^{\alpha}} \inf \left\{I(a): a<\mu-\varepsilon^{\prime}\right\} \\
& =-\infty,
\end{aligned}
$$

as $I(a)=\infty$ for all $a<\mu$.

If $x \in\left(0, \frac{1}{2}\right)$ then $\bar{x}=1-x$ and we apply a similar argument as in the $x=0$ case, but starting with the following set inclusions:

$$
\left\{\frac{S_{N_{t}}^{\xi}}{t} \in B_{\varepsilon}(x), \frac{N_{t}}{t}>\frac{x}{\mu-\varepsilon^{\prime}}\right\} \subset\left\{\frac{S_{N_{t}}^{\xi}}{t}<x+\varepsilon, \frac{N_{t}}{t}>\frac{x}{\mu-\varepsilon^{\prime}}\right\} \subset\left\{S_{\left\lceil t x / \mu-\varepsilon^{\prime}\right\rceil}^{\xi}<(x+\varepsilon) t\right\} .
$$

Applying the large deviations upper bound from Theorem 1 for this final sequence of sets, we have

$$
\begin{aligned}
\limsup _{t \rightarrow \infty} \frac{1}{t^{\alpha}} \log \mathrm{P}\left(S_{\left\lceil t x /\left(\mu-\varepsilon^{\prime}\right)\right\rceil}^{\xi}<(x+\varepsilon) t\right) & \leq-\frac{x^{\alpha}}{\left(\mu-\varepsilon^{\prime}\right)^{\alpha}} \inf \left\{I(a): a<\left(\mu-\varepsilon^{\prime}\right)\left(1+\frac{\varepsilon}{x}\right)\right\} \\
& =-\frac{x^{\alpha}}{\left(\mu-\varepsilon^{\prime}\right)^{\alpha}} I\left(\left(\mu-\varepsilon^{\prime}\right)\left(1+\frac{\varepsilon}{x}\right)\right) .
\end{aligned}
$$

As $\varepsilon$ tends to 0 , the $I$ argument is strictly less than $\mu$ and $I(a)=\infty$ for all $a<\mu$. Thus, for all $0<\varepsilon^{\prime}<\mu$, in the limit as $\varepsilon$ tends to 0 , the right-hand side equals $-\infty$.

When $x \in\left(\frac{1}{2}, 1\right]$, we use the fact that $T_{N_{t}}=S_{N_{t}}^{\xi}+S_{N_{t}}^{\tau} \leq t$ to give us the set inequality

$$
\left\{\frac{S_{N_{t}}^{\xi}}{t}>x-\varepsilon\right\} \subset\left\{\frac{S_{N_{t}}^{\tau}}{t}<1-x+\varepsilon\right\} \text {. }
$$


If $x \in\left(\frac{1}{2}, 1\right)$ then $\bar{x}=x$ and we have the set inclusions

$$
\begin{aligned}
\left\{\frac{S_{N_{t}}^{\xi}}{t} \in B_{\varepsilon}(x), \frac{N_{t}}{t}>\frac{1-x}{\mu-\varepsilon^{\prime}}\right\} & \subset\left\{\frac{S_{N_{t}}^{\xi}}{t}>x-\varepsilon, \frac{N_{t}}{t}>\frac{1-x}{\mu-\varepsilon^{\prime}}\right\} \\
& \subset\left\{\frac{S_{N_{t}}^{\tau}}{t}<(1-x+\varepsilon), \frac{N_{t}}{t}>\frac{1-x}{\mu-\varepsilon^{\prime}}\right\} \\
& \subset\left\{S_{\left\lceil t(1-x) /\left(\mu-\varepsilon^{\prime}\right)\right\rceil}^{\tau}<(1-x+\varepsilon) t\right\} .
\end{aligned}
$$

Again we apply the large deviations upper bound from Theorem 1 for this final sequence of sets and take the limit $\varepsilon$ tends to 0 , which gives a rate of $-\infty$. When $x=1$, we have

$$
\begin{aligned}
\left\{\frac{S_{N_{t}}^{\xi}}{t} \in B_{\varepsilon}(1), \frac{N_{t}}{t}>\frac{1-x}{\mu-\varepsilon^{\prime}}\right\} & \subset\left\{\frac{S_{N_{t}}^{\xi}}{t}>1-\varepsilon, \frac{N_{t}}{t}>\frac{1-x}{\mu-\varepsilon^{\prime}}\right\} \\
& \subset\left\{\frac{S_{N_{t}}^{\tau}}{t}<\varepsilon, \frac{N_{t}}{t}>\frac{\varepsilon}{\mu-\varepsilon^{\prime}}\right\} \\
& \subset\left\{S_{\left\lceil t \varepsilon /\left(\mu-\varepsilon^{\prime}\right)\right\rceil}^{\tau}<\varepsilon t\right\},
\end{aligned}
$$

and the result follows as in the $x=0$ case.

Lemma 2. (Small n.) For $x \in(0,1)$, define $\bar{x}:=\max (x, 1-x)$. For any $x \in(0,1)$, we have

$$
\lim _{\varepsilon \rightarrow 0} \lim _{\varepsilon^{\prime} \rightarrow 0} \limsup _{t \rightarrow \infty} \frac{1}{t^{\alpha}} \log \mathrm{P}\left(\frac{S_{N_{t}}^{\xi}}{t} \in B_{\varepsilon}(x), \frac{N_{t}}{t} \leq \frac{1-\bar{x}}{\mu-\varepsilon^{\prime}}\right) \leq-J(x) .
$$

If $x=0$ or $x=1$, we have

$$
\lim _{\varepsilon \rightarrow 0} \lim _{\varepsilon^{\prime} \rightarrow 0} \limsup _{t \rightarrow \infty} \frac{1}{t^{\alpha}} \log \mathrm{P}\left(\frac{S_{N_{t}}^{\xi}}{t} \in B_{\varepsilon}(x), \frac{N_{t}}{t} \leq \frac{\varepsilon}{\mu-\varepsilon^{\prime}}\right) \leq-J(x) .
$$

Proof. Throughout let $0<\varepsilon^{\prime}<\mu$. Consider $x=1$. As $S_{n}^{\xi}$ is increasing in $n$,

$$
\left\{\frac{S_{N_{t}}^{\xi}}{t} \in B_{\varepsilon}(1), \frac{N_{t}}{t} \leq \frac{\varepsilon}{\mu-\varepsilon^{\prime}}\right\}=\left\{\frac{S_{N_{t}}^{\xi}}{t}>1-\varepsilon, \frac{N_{t}}{t} \leq \frac{\varepsilon}{\mu-\varepsilon^{\prime}}\right\} \subset\left\{S_{\left\lceil\varepsilon t /\left(\mu-\varepsilon^{\prime}\right)\right\rceil}^{\xi}>(1-\varepsilon) t\right\} .
$$

Using the large deviations upper bound from Theorem 1 on this final sequence of sets, we obtain

$$
\begin{aligned}
\limsup _{t \rightarrow \infty} & \frac{1}{t^{\alpha}} \log \mathrm{P}\left(S_{\left\lceil\varepsilon t /\left(\mu-\varepsilon^{\prime}\right)\right\rceil}^{\xi}>(1-\varepsilon) t\right) \\
& =\frac{\varepsilon^{\alpha}}{\left(\mu-\varepsilon^{\prime}\right)^{\alpha}} \limsup _{n \rightarrow \infty} \frac{1}{n^{\alpha}} \log \mathrm{P}\left(\frac{S_{n}^{\xi}}{n}>(1-\varepsilon) \frac{\mu-\varepsilon^{\prime}}{\varepsilon}\right) \\
& \leq-\frac{\varepsilon^{\alpha}}{\left(\mu-\varepsilon^{\prime}\right)^{\alpha}} I\left(\frac{(1-\varepsilon)\left(\mu-\varepsilon^{\prime}\right)}{\varepsilon}\right) \\
& =-\left(1-\varepsilon\left(1+\frac{\mu}{\mu-\varepsilon^{\prime}}\right)\right)^{\alpha}
\end{aligned}
$$

and the result follows by letting $\varepsilon^{\prime}$ tend to 0 and then letting $\varepsilon$ tend to 0 . 
Next consider $x \in\left(\frac{1}{2}, 1\right)$, so that $\bar{x}=x$. With $n_{t}:=\left\lceil t(1-\bar{x}) /\left(\mu-\varepsilon^{\prime}\right)\right\rceil$, we have

$$
\left\{\frac{S_{N_{t}}^{\xi}}{t} \in B_{\varepsilon}(x), \frac{N_{t}}{t} \leq \frac{1-\bar{x}}{\mu-\varepsilon^{\prime}}\right\} \subset\left\{\frac{S_{N_{t}}^{\xi}}{t}>x-\varepsilon, \frac{N_{t}}{t} \leq \frac{1-\bar{x}}{\mu-\varepsilon^{\prime}}\right\} \subset\left\{\frac{S_{n_{t}}^{\xi}}{t}>x-\varepsilon\right\} .
$$

Now using the large deviations upper bound from Theorem 1, we have

$$
\begin{aligned}
\limsup _{t \rightarrow \infty} \frac{1}{t^{\alpha}} \log \mathrm{P}\left(\frac{S_{n_{t}}^{\xi}}{t}>x-\varepsilon\right) & =\frac{(1-x)^{\alpha}}{\left(\mu-\varepsilon^{\prime}\right)^{\alpha}} \limsup _{n \rightarrow \infty} \frac{1}{n^{\alpha}} \log \mathrm{P}\left(\frac{S_{n}^{\xi}}{n}>\frac{(x-\varepsilon)\left(\mu-\varepsilon^{\prime}\right)}{1-x}\right) \\
& \leq-\frac{(1-x)^{\alpha}}{\left(\mu-\varepsilon^{\prime}\right)^{\alpha}} \inf \left\{I(a): a>\frac{(x-\varepsilon)\left(\mu-\varepsilon^{\prime}\right)}{1-x}\right\} \\
& =-\frac{(1-x)^{\alpha}}{\left(\mu-\varepsilon^{\prime}\right)^{\alpha}} I\left(\frac{(x-\varepsilon)\left(\mu-\varepsilon^{\prime}\right)}{1-x}\right) \\
& =-\left(x-\varepsilon+(x-1) \frac{\mu}{\mu-\varepsilon^{\prime}}\right)^{\alpha} .
\end{aligned}
$$

Thus, the upper bound follows by letting $\varepsilon^{\prime}$ tend to 0 and then letting $\varepsilon$ tend to 0 .

The results for $x \in\left[0, \frac{1}{2}\right.$ ) follow analogously using the corresponding constraints on $S_{N_{t}}^{\tau}$ : for $x=0$,

$$
\left\{\frac{S_{N_{t}}^{\xi}}{t} \in B_{\varepsilon}(0), \frac{N_{t}}{t} \leq \frac{\varepsilon}{\mu-\varepsilon^{\prime}}\right\} \subset\left\{S_{\left\lceil\varepsilon t /\left(\mu-\varepsilon^{\prime}\right)\right\rceil}^{\tau}>(1-\varepsilon) t\right\},
$$

and, for $x \in\left(0, \frac{1}{2}\right)$,

$$
\left\{\frac{S_{N_{t}}^{\xi}}{t} \in B_{\varepsilon}(0), \frac{N_{t}}{t} \leq \frac{\varepsilon}{\mu-\varepsilon^{\prime}}\right\} \subset\left\{\frac{S_{n_{t}}^{\tau}}{t}>x-\varepsilon\right\} .
$$

With $x \in(0,1)$, for any $0<\varepsilon^{\prime}<\mu$, by the principle of the largest term (see, e.g. [2, Lemma 1.2.15]),

$$
\begin{aligned}
\limsup _{t \rightarrow \infty} \frac{1}{t^{\alpha}} \log \mathrm{P}\left(\frac{S_{N_{t}}^{\xi}}{t} \in B_{\varepsilon}(x)\right)= & \max \left(\limsup _{t \rightarrow \infty} \frac{1}{t^{\alpha}} \log \mathrm{P}\left(\frac{S_{N_{t}}^{\xi}}{t} \in B_{\varepsilon}(x), \frac{N_{t}}{t}>\frac{1-\bar{x}}{\mu-\varepsilon^{\prime}}\right),\right. \\
& \left.\limsup _{t \rightarrow \infty} \frac{1}{t^{\alpha}} \log \mathrm{P}\left(\frac{S_{N_{t}}^{\xi}}{t} \in B_{\varepsilon}(x), \frac{N_{t}}{t} \leq \frac{1-\bar{x}}{\mu-\varepsilon^{\prime}}\right)\right) \\
= & \limsup _{t \rightarrow \infty} \frac{1}{t^{\alpha}} \log \mathrm{P}\left(\frac{S_{N_{t}}^{\xi}}{t} \in B_{\varepsilon}(x), \frac{N_{t}}{t} \leq \frac{1-\bar{x}}{\mu-\varepsilon^{\prime}}\right),
\end{aligned}
$$

where the last line follows as Lemma 1 proves that the first term in the max is $-\infty$. As this is true for all $0<\varepsilon^{\prime}<\mu$, the following upper bound follows from Lemma 2 after letting $\varepsilon^{\prime}$ tend to 0 and then letting $\varepsilon$ tend to 0 :

$$
\lim _{\varepsilon \rightarrow 0} \limsup _{t \rightarrow \infty} \frac{1}{t^{\alpha}} \log \mathrm{P}\left(\frac{S_{N_{t}}^{\xi}}{t} \in B_{\varepsilon}(x)\right) \leq-J(x) .
$$

A near identical application of the lemmas suffices for $x=0$ and $x=1$. Thus, as the lower and upper deviation functions for $\left\{S_{N_{t}}^{\xi} / t\right\}$ coincide with $-J(\cdot)$, Proposition 1 is proved. 


\section{Acknowledgements}

This work was supported by Science Foundation Ireland. K. Duffy was supported by grant number IN3/03/I346. When this work began, A. Sapozhnikov was working for the Department of Mathematics and Boole Centre for Research in Informatics, University College Cork, Ireland, and was supported by grant number SFI-04-RP1-I512. He thanks the Hamilton Institute for hospitality during the writing of this paper.

The authors thank an anonymous referee for their careful reading of the article and for helpful comments on the proof's structure that enabled us to significantly improve its presentation.

\section{References}

[1] Bryc, W. and Dembo, A. (1996). Large deviations and strong mixing. Ann. Inst. H. Poincaré Prob. Statist. 32, 549-569.

[2] Dembo, A. and Zeitouni, O. (1998). Large Deviation Techniques and Applications. Springer, Berlin.

[3] Gantert, N. (1998). Functional Erdős-Renyi laws for semiexponential random variables. Ann. Prob. 26, 13561369.

[4] Nagaev, S. V. (1979). Large deviations of sums of independent random variables. Ann. Prob. 7, 745-789. 